\title{
Monitoring and Protection Method of a REBCO Magnet Designed for Electromagnetic Vibration Tests
}

\begin{abstract}
Katsutoshi MIZUNO
Minoru TANAKA

Masafumi OGATA

Cryogenic Systems Laboratory, Maglev Systems Technology Division

REBCO (Rare-Earth Barium Copper Oxide) is a high temperature superconducting material and is suitable for use in on-board maglev train magnets because of its high current density in a high magnetic field. A real-scale REBCO coil was fabricated and its basic performance confirmed. As a next step in this research, electromagnetic vibration tests on ground coils using real-scale REBCO coils are planned. Since electromagnetic vibration tests usually take several weeks to complete, a monitoring and protection system for the REBCO magnet is required. A high-sensitive coil voltage detection method was evaluated.
\end{abstract}

Keywords: $R E B C O$, electromagnetic vibration, protection, thermal runaway

\section{Introduction}

REBCO (Rare-Earth Barium Copper Oxide) is one among a number of high temperature superconducting (HTS) materials. Many applications using this material have been developed (e.g. MRI, NMR, and motor/generators). REBCO coated conductors are superior to other HTS wires in terms of current density in high magnetic fields. Therefore, REBCO superconducting wire can reach a high operating temperature that cannot be achieved with other superconducting wires. At RTRI, REBCO magnets have been developed with a view to applying them to the maglev system. A real-scale REBCO coil has already been built, and confirmation has been obtained that it can produce the $700 \mathrm{kA}$ magnetomotive force required for a maglev system [1]. (Magnetomotive force is the product of the number of coil turns and the excitation current.) In addition, mechanical vibration tests assuming actual running conditions have been carried out. Stable excitation of the REBCO magnet under vibration conditions was confirmed [2].

In order to adapt the REBCO magnet for use with maglev, monitoring and protection methods are essential for stable operation of the magnet in addition to fine-tuning of the REBCO magnet design. If a part of the superconductor transitions from a superconducting state to a normal conduction state (hereafter, referred to as transition from sc to nc) occurs due to a malfunction, the normal conduction area expands due to Joule heating, and eventually the entire coil generates heat and the superconducting magnet function is lost. This phenomenon is called a quench. It is essential to avoid quench and to protect the superconducting magnet from damage if a quench should occur. Since the cooling method and the physical properties (specific heat, thermal conductivity and so on) of the structural members of a high temperature superconducting magnet are very different to a low temperature superconducting magnet, quench countermeasures are also different. Therefore, in HTS magnets, quench is referred to a "thermal runaway" (for details, see the section 2.1). Furthermore, even among HTS magnets, differences in current density, stored energy and operating temperature will also affect how difficult it is to protect them. Consequently, it is not possible to develop a turn-key monitoring and protection method: each approach has to be tailored to each application.

The next step in this research will be to conduct electromagnetic vibration tests on a real-scale REBCO magnet that had been built. Electromagnetic vibration tests are important for testing the endurance of ground coils used for maglev. The superconducting magnet used for this test has to have the same specifications as an actual on-board magnet, and be tested for a maximum operating time of up to several weeks. Therefore, these electromagnetic vibration tests were also meaningful for the development of the REBCO magnet. This paper describes the development of a monitoring and protecting method for REBCO magnets.

\section{Protection of a REBCO coil}

The innovative approach adopted for REBCO coil protection is to use non-insulated coil [3]. Even without insulation between turns, contact resistance exists between them. Because of the zero-electric resistance of the superconductor, in superconducting steady state the current goes through the conductor and there is no bypass between turns. However, when sc transitions to nc, there is a bypass of the current through the turns, which protects the coil. Although this coil design would be effective as a protection for the coil, there are some problems.

The bypassing current during magnetization or demagnetization, means it takes a long time for the magnet to enter into operation. Joule heating due to the bypass current can be also a problem depending on the coil specifications and the cooling method.

Although this insulation method could effective as a coil protection, it is unclear for which coil specifications it would be effective. In a large REBCO magnet of the kind used with maglev, these problems are exacerbated, which rules out application of the non-insulation method. This research therefore examines methods for protecting REBCO coils, with insulation.

\subsection{Concept of protection}

When quench is confirmed in the superconducting 
magnet, the basic idea of coil protection is that the excitation power is disconnected by a circuit breaker, and the energy stored in the coil is consumed by the protective resistance (see Fig.1). However, this quench protection would not work in REBCO magnets. In simple terms, the specific heat increases significantly at high temperature. For example, the specific heat of copper is $58 \mathrm{~J} /(\mathrm{K} \cdot \mathrm{kg})$ at $40 \mathrm{~K}$ while it is only $0.1 \mathrm{~J} /(\mathrm{K} \cdot \mathrm{kg})$ at $4 \mathrm{~K}$. Therefore, in a high temperature superconducting magnet, a transition from sc to nc would not propagate to the entire coil in a short time. When the cooling balance is lost, the REBCO coated conductor may burn-out even with a small amount of Joule heating. This phenomenon is called thermal runaway which makes it difficult to protect HTS.

If the thermal runaway begins, even if the power supply is stopped, some of the energy stored in the coils is consumed not in the protective resistance but in the normal conducting area inside the coil. A large coil with a large amount of stored energy has a higher risk of burn-out. This Joule heating in the hot spot also depends on the value of the protective resistance. A large resistance value means fast dumping which is effective in reducing the Joule heating time in the hot spot. However, the peak voltage of the coil is decided by the product of the operating current and the value of the protective resistance. There is an upper limit of resistance based on the withstanding voltage of the coil.

Considering these conditions, the most reliable protection method is to shut down power or reduce the current before thermal runaway occurs. In other words, the key to protecting the REBCO magnet protection lies in being able to achieve the following:

1. Detection of phenomena causing the transition from sc to nc

2. Detection of transition from sc to nc before the cooling balance is lost

Three factors determine whether a superconducting wire will maintain its superconducting state, or whether sc to nc transition will occur: magnetic field, current and temperature. In maglev, the magnetic field generated by the ground coils is relatively small compared to the self-produced magnetic field of the on-board superconducting mag-

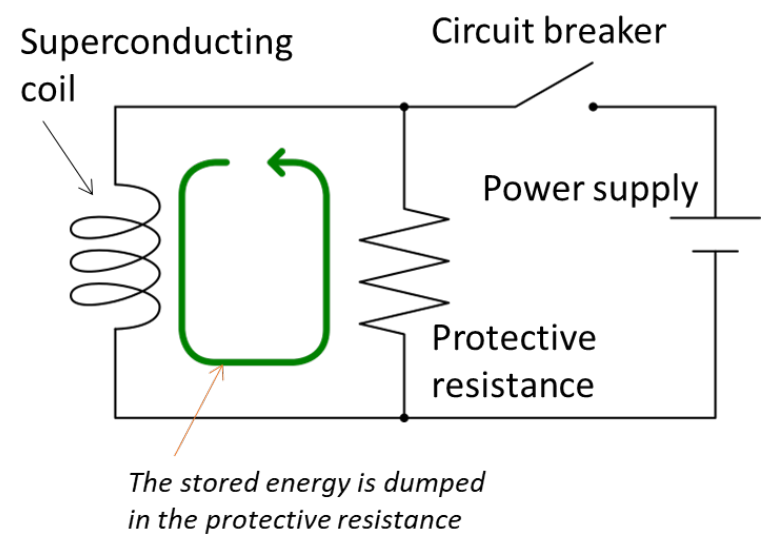

Fig. 1 Example of a protection circuit for a superconducting magnet net. Since most varying magnetic fields are shielded by an outer vessel or coil case, the possibility of sc to nc transition occurring because of induced current due to variation in the magnetic field in the ground coils is small. Magnetic field and current are stable during magnet operation. However, coil temperature may vary for a number of reasons. For example, if cooling capacity decreases over long-term operation, or if an unpredictable cryocooler failure occurs. There is also the possibility of heat load increasing because of a reduction in a degree of vacuum in the magnet.

Early detection of sc to nc transition can be achieved through coil voltage. In a large REBCO coil, since the power supply voltage ripple or noise from environmental magnetic field increases, it is necessary to be able to accurately measure voltage given this noisy environment.

\subsection{Possibility of voltage monitoring}

Since a large number of superconducting magnets must be monitored in a maglev system, it is necessary to have a low-cost high reliability monitoring method. Consequently, the high-accuracy voltage measurement system must be built with general-purpose measuring equipment and a simplified configuration.

This section reviews the voltage fluctuation test results from a real-scale REBCO coil under excitation [1]. The real scale coil consists of 8 pancake coils as shown in Fig. 2. The voltage of each of the eight coils was measured as well as the total coil voltage. Figure 3 shows the voltage fluctua-

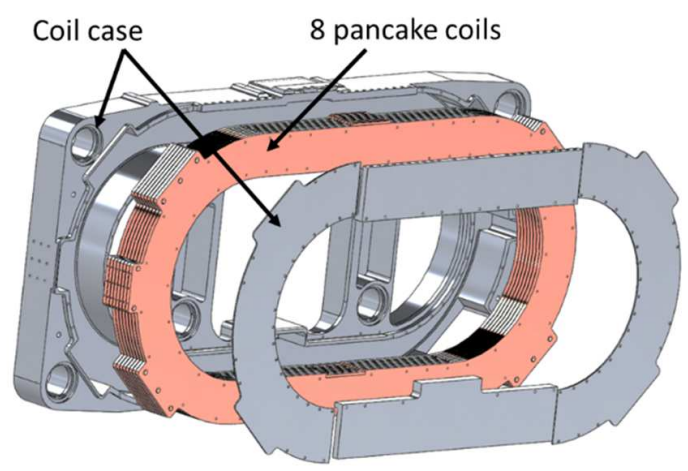

Fig. 2 Inner structure of the real-scale REBCO coil [4]

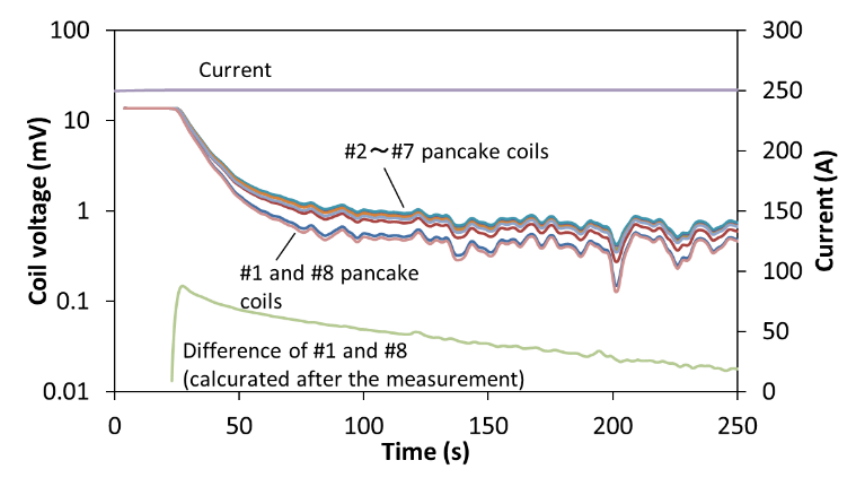

Fig. 3 Voltage behavior of the real-scale REBCO coil during rated excitation 
tion of the eight pancake coils at the excitation current of $250 \mathrm{~A}$ (700 kA magnetomotive force).

Regardless of the stable current, the coil voltages were unstable and gradually decreased. This is the effect of the shielding current (loop current inside the super conducting layer of the conductor). It is notable that the noise voltage of each of eight coils had the same pattern. The distribution of the shielding current of the coils at the symmetrical positions was assumed to be similar, e.g., the uppermost \#1 and the lowermost \#8, and the voltage attenuation was the same. Figure 3 shows the voltage difference between \#1 and \#8 that was calculated after the measurement. The noise was almost removed and the voltage decay due to the shielding current was one tenth of the raw data.

If the coil voltage differences are calculated in real time, then it should be possible to detect sc to nc transition with a high degree of accuracy based on small coil voltage. When the transition occurs in the paired pancake coils at the same time, the coil voltage difference remains at zero and transition may not be detected. However, this situation is rare since the performance of the REBCO coated conductor is not uniform and each coil has a different upper limit temperature. Although coil voltage measurement using the bridge circuit has a similar effect, only continuous pancake coils can be paired such as \#1 and \#2. In the calculation of the coil voltage difference, any pancake coil can be paired and no extra circuit is necessary. Simple calculation by microcomputer, PLC or laptop means it is possible to detect sc to nc transition with a high degree of accuracy.

\section{Protection tests with small coils}

Even if detection of sc to nc transition based on coil voltage difference is effective, the detection voltage must be lower than the voltage at which the cooling balance is lost. However, the threshold value at which the cooling balance is lost is unknown.

Therefore, to get fundamental data, thermal runaway tests were carried out with small coils [5].

\subsection{Small coil specifications}

As thermal runaway of HTS coils is a local phenomenon, the behavior of the coil voltage is considered to depend on the cooling method and coil structure rather than the coil size. The small coils in this experiment had the same structure as the real-scale coil; cooling members were bonded to the upper and lower surfaces of the coil winding by thermoplastic resin. Two small coils were used in this experiment to evaluate the difference of the cooling member materials. One was made of GFRP, and the other was made by aligning copper wires and hardening them with resin. The specifications of the small coils are summarized in Table 1, and Fig. 4 shows a picture of a coil with the copper cooling member.

\subsection{Coil voltage behavior during the thermal run- away}

In the experiment, the small coil was cooled by a cryocooler and excited at $160 \mathrm{~A}$. Then, the cryocooler was
Table 1 Specifications of small coils used in thermal runaway tests

\begin{tabular}{|l|l|}
\hline Coil shape & $\begin{array}{l}\text { Double pancake coil } \\
\text { Inner diameter: } 60 \mathrm{~mm} \\
\text { Outer diameter: } 92 \mathrm{~mm}\end{array}$ \\
\hline Number of turns & $124 \times 2$ \\
\hline REBCO coated conductor & $\begin{array}{l}\text { SuperPower Inc., } \\
\text { SCS6050-AP }\end{array}$ \\
\hline Total length of the conductor & $60 \mathrm{~m}$ \\
\hline Inductance & $5 \mathrm{mH}$ \\
\hline
\end{tabular}

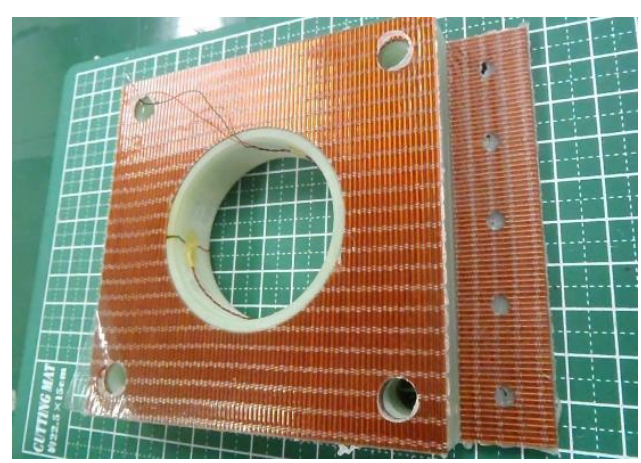

Fig. 4 Small coil for thermal runaway tests. The cooling members on the surface are made of aligned copper wires and hardened with resin

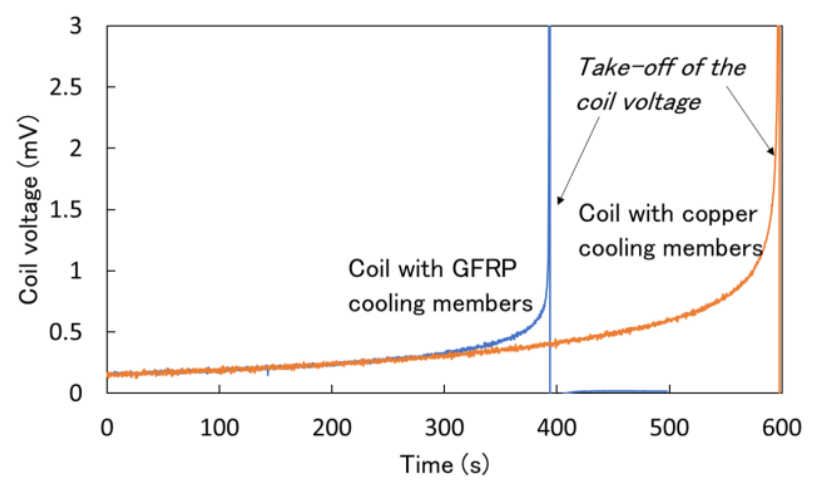

Fig. 5 Voltage behavior of small coils before thermal runaway

stopped to raise the coil temperature gradually while the current was kept at $160 \mathrm{~A}$. When the coil temperature reached $65 \mathrm{~K}$, the voltage was observed and measuring was continued until a voltage jump occurred (hereafter, referred to as 'take-off'). The coil voltage behavior before take-off is shown in Fig. 5. The origin of the X-axis in Fig. 5 is set at the coil voltage of $0.15 \mathrm{mV}$. The voltage in both small coils increased at the same rate to begin with. However, at around $0.3 \mathrm{mV}$, the voltage of the small coil with GFRP cooling members increased rapidly. Finally, it jumped at around $0.7 \mathrm{mV}$. On the other hand, the voltage increase rate in the small coil with copper cooling members remained low and the take-off voltage was above $1 \mathrm{mV}$. Understandably, the copper cooling member has a higher thermal conductivity. As the result, heat due to the transition spreads out and the take-off voltage is higher.

These results indicate that high-speed detection is not necessary, because the increase in voltage is slow be- 
fore take-off. For example, if the detection voltage is 0.5 $\mathrm{mV}$, this leaves over 20 seconds before take-off even in the GFRP cooling member coil, which is sufficient time for power to be cut.

\subsection{Demonstration of a protection system}

As the required voltage detection level and detection time were clarified, as described in 3.2 , a protection system was constructed to demonstrate the availability of monitoring of the coil voltage difference. Although this paper only describes the monitoring of the coil voltage difference, the protection system also has temperature and vacuum monitoring. Figure 6 shows the basic constitution of the protection system. All the components in Fig. 6 are commercially available and not customized: a multi-channel data logger by GRAPHTEC Corp., temperature monitors by Lake Shore Cryotronics Inc., a switching power supply by Takasago International Corp. and a control software LabVIEW by National Instruments. Each component is connected by serial port or USB. As mentioned in section 3.2, extremely high speed detection and power shutdown are not necessary and these communication methods satisfy the system requirements. Incidentally, the circuit breaker

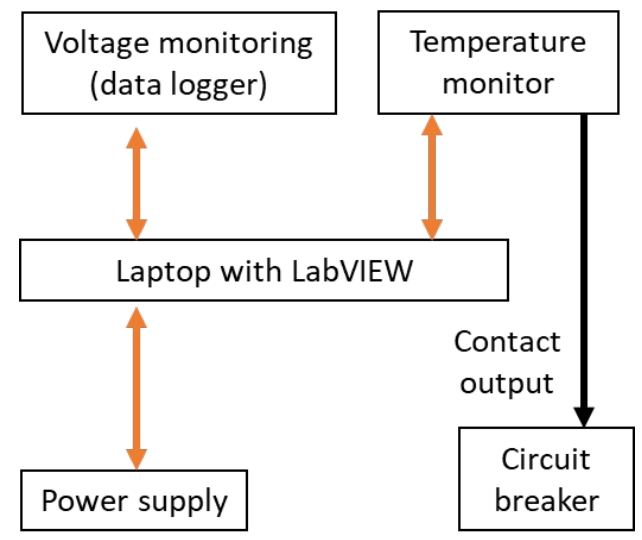

Fig. 6 Basic constitution of protection system works by contact output of the temperature monitor since the laptop with LabVIEW does not have a contact output.

In the protection test, as well as the previous test in 3.2 , thermal runaway was caused intentionally. The small coil was also used in this experiment. The small coil was a double pancake coil with two layers of winding (upper coil and lower coil). Figure 7 shows the coil voltages from the beginning of the excitation to the power shutdown. During the current sweeping, the upper and lower coils had high voltages because of their inductances. However, both coil were identical and the induced voltages were the same. Hence the induced voltages were canceled and coil voltage differences were maintained zero. In other words, this protection system works during current sweeps.

When the current reached $160 \mathrm{~A}$, the upper coil already showed the voltage. Finally, the upper coil voltage took off at around 600 seconds. As voltage increased, the noise patterns were similar. It is obvious that the coil voltage detection level was improved because of the difference in the upper and lower coils voltages. Voltage detection below $0.1 \mathrm{mV}$ was easily achieved in this small coil. (Note, the detection voltage was set at $12.5 \mathrm{mV}$ in this experiment to observe thermal runaway.)

\section{REBCO magnet for the electromagnetic vibration tests}

The real-scale REBCO coil was installed in a cryostat specially designed for the electromagnetic vibration tests in parallel with the construction of the protection system. The features of this magnet and noise level during the excitation are explained in this chapter.

\subsection{Electromagnetic vibration tests}

Electromagnetic vibration tests are one of a series of endurance tests conducted on maglev ground coils. Ground coils are arranged in front of the superconducting magnet. Then, the ground coils are energized using AC power in a high magnetic field generated by the superconducting mag-

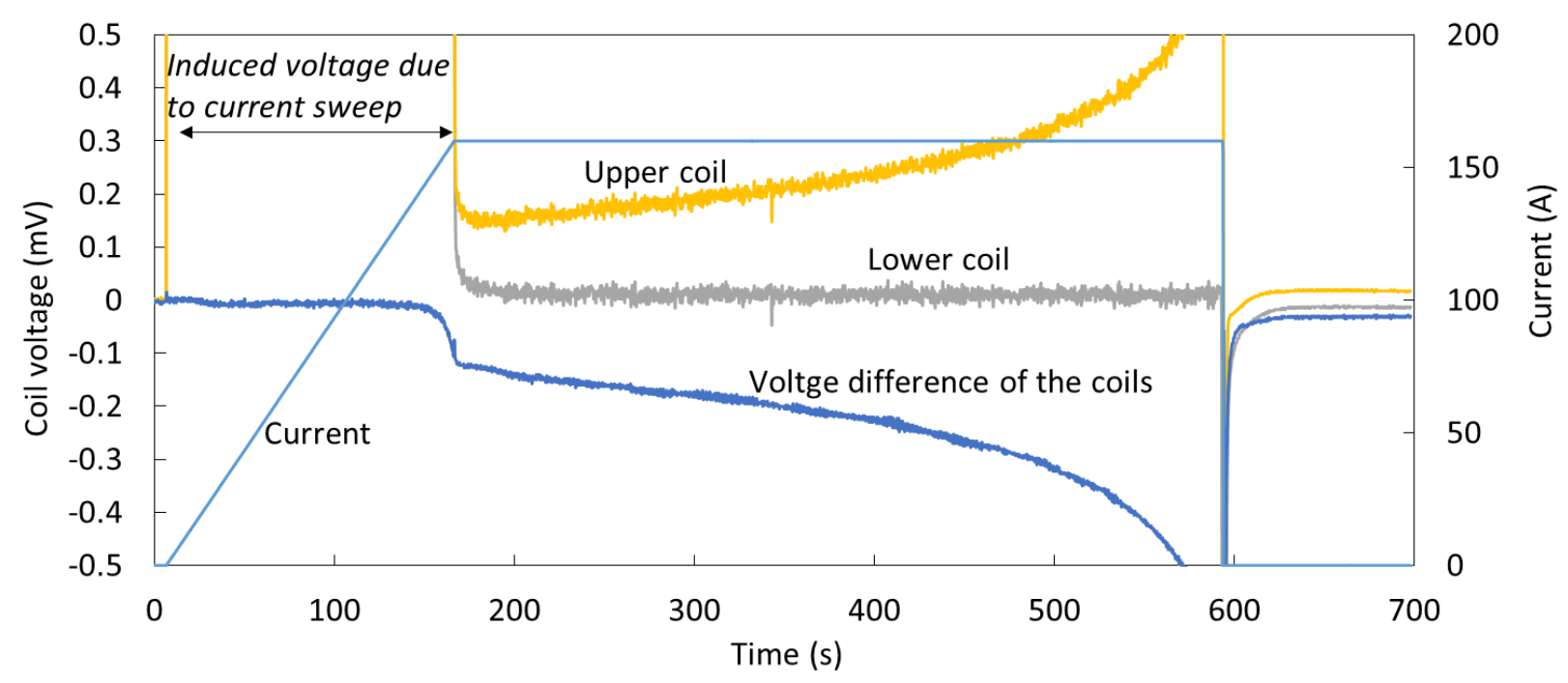

Fig. 7 Measurements of voltage differences in small coil 
net (see Fig. 8). The vibration mechanism is basically same as actual running vibrations. For the vibration tests, an actual on-board magnet or a magnet with the same dimensions and the magnetomotive force is necessary. Until today, low temperature superconducting magnets have been used for these tests. If these magnets are replaced with REBCO magnets, cryogens such as liquid nitrogen and liquid helium would be unnecessary. Cooling time would also be shortened. Hence, electromagnetic vibration tests could be carried out efficiently at a lower cost.

Electromagnetic vibration tests are also important for the REBCO magnet. It is now possible to conduct tests that vibrate not only the REBCO coil but also whole the mag-

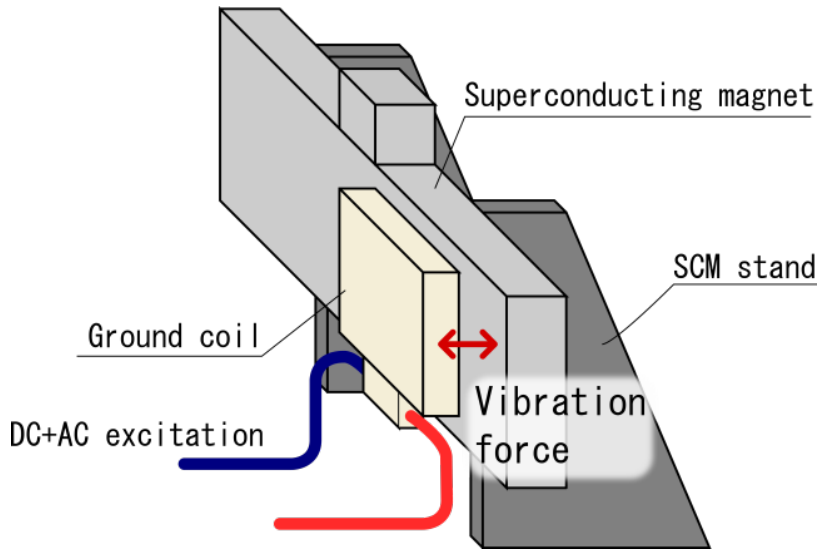

Fig. 8 Setup of electromagnetic vibration test

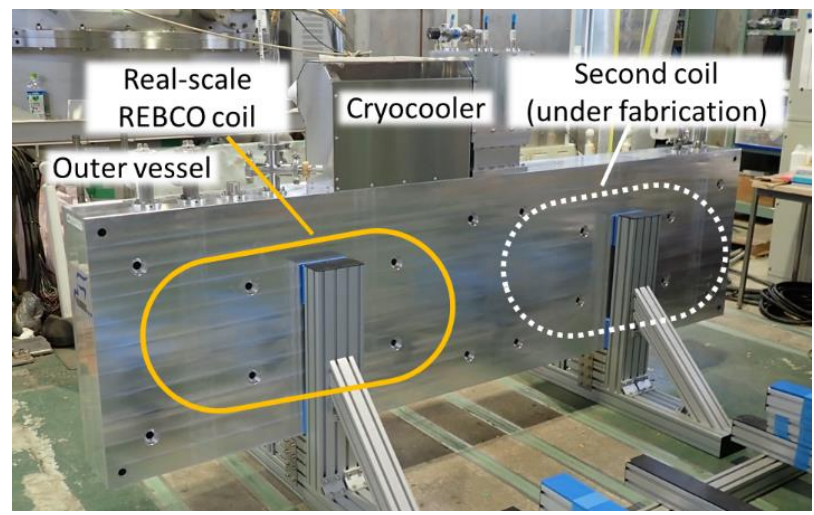

Fig. 9 REBCO magnet for electromagnetic vibration tests net including the cryocooler and the outer vessel. In the mechanical vibration tests, the REBCO coil was suspended from the top flange of the cryostat and vibrated freely. However, the Eigen modes and Eigen frequencies of the coil depend on how the magnet is fixed. Therefore, electromagnetic vibration tests provide more accurate vibration characteristics of the REBCO magnet. Moreover, long-term vibration is possible because of the absence of mechanical moving parts. Long-term operational data for the REBCO magnet could therefore also be gathered.

\subsection{Features of the REBCO magnet}

As mentioned above, the outer vessel (vacuum vessel) must have almost the same outer dimension as the actual on-board magnet. In particular, the distance between the ground coils to the REBCO coil is important. The outer vessel was redesigned based on the cooling method and insulation structure. Although the on-board magnet consists of 4 superconducting coils, 2 coils are sufficient for vibration tests. The outer vessel for the REBCO magnet is also a two-coil design (see Fig. 9), and the second coil is being built. Since some types of ground coil (levitation and guidance coil) can be tested with one REBCO coil, the electromagnetic vibration test will start with the one-coil REBCO magnet.

\subsection{Excitation confirmation}

The one-coil REBCO magnet was assembled and excited. The REBCO coil was cooled at around $30 \mathrm{~K}$ by the cryocooler. The excitation current was $250 \mathrm{~A}$ (equivalent to $700 \mathrm{kA}$ magnetomotive force) and the magnet was maintained in the excitation state for one hour. The measuring conditions are as follows: The sampling rate is $250 \mathrm{~ms}$. The voltage measurement range is $100 \mathrm{mV}$. The LPF is $1.5 \mathrm{~Hz}$. All the pancake coils (\#1 to \#8) voltages were measured simultaneously. As the representative data, the coil voltage of coils \#1 and \#8 and their voltage difference are shown in Fig. 10. The protection system was used in this measurement and the difference was calculated in real time. The inductance of the REBCO magnet was $12 \mathrm{H}$, while that of the small coil was only $5 \mathrm{mH}$. The noise was much larger than with the small coils because of the large inductance.

The voltage behaviors of coils \#1 and \#8 were quite similar as expected. The amplitude of the voltage differ-

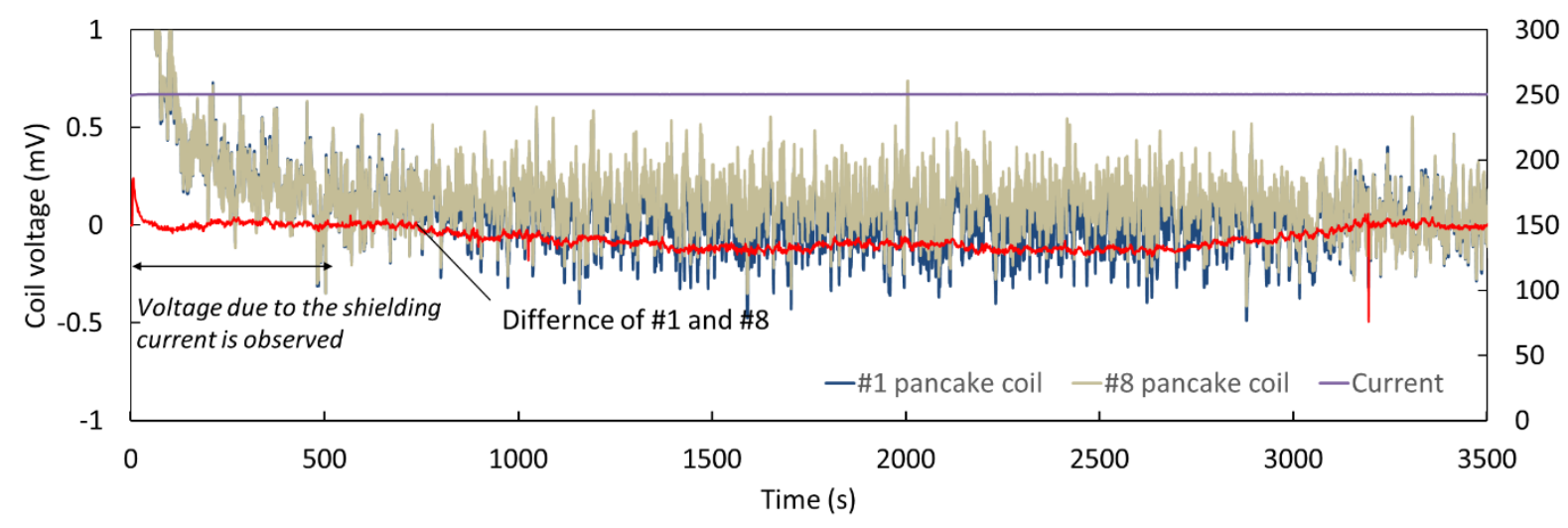

Fig. 10 Voltage behavior of REBCO magnet during rated excitation 
ence including long-term variation was only $0.1 \mathrm{mV}$, while the original noise amplitude was $\pm 0.5 \mathrm{mV}$. Although more than 600 seconds were needed until the shielding current disappeared, the voltage difference stabilized in less than 60 seconds. Since high speed detection and power shutdown are not necessary, only the continuous high voltage is used to detect the transition in this protection system. An unexpected voltage spike at 3200 seconds was not interpreted as a false detection.

Since the coil temperature and the excitation currents were different to those in the small coil tests, the precise take-off voltage was unclear. (Thermal runaway tests on the real-scale coil carries the risk of burn out, and damaging the REBCO coil before the electromagnetic vibration tests is not acceptable.) However, it is quite unlikely that these small differences in conditions reduce the take-off voltage drastically. For this reason, the monitoring of the voltage difference is also considered to be effective for detecting sc to nc transition in real-scale REBCO coils.

\section{Conclusion}

With the prospect of electromagnetic vibration tests on a REBCO magnet, a monitoring and protection system for the magnet was developed and tested. The REBCO coil consisted of several pancake coils. The transition before loss of cooling balance was detected by monitoring voltage differences in pancake coils in symmetric position.

A detection level of $0.1 \mathrm{mV}$ was achieved in the REBCO magnet. This monitoring system is considered highly effective to protect REBCO magnets from the thermal runaway.

The monitoring and protection system is planned to be enhanced by combining voltage difference monitoring with temperature and the vacuum monitoring. Electromagnetic vibration tests on the REBCO coil are also planned.

\section{Acknowledgement}

This work was financially supported by the Japanese Ministry of Land, Infrastructure, Transport and Tourism.

\section{References}

[1] Mizuno K., Sugino M., Tanaka M. and Ogata M., "Experimental Production of a Real-Scale REBCO Magnet Aimed at Its Application to Maglev," IEEE Trans. Appl. Supercond., Vol. 27, 3600205, 2017.

[2] Mizuno K., Tanaka M., Ogata M. and Okamura T., "Mechanical Vibration Test of a REBCO Coil Designed for Application to the Maglev," IEEE Trans. Appl. Super-cond., Vol. 28, Issue 4, 3601107, 2018.

[3] S. Hahn, D. K. Park, J. Bascunan and Y. Iwasa, "HTS Pancake Coils Without Turn-to-Turn Insulation," IEEE Trans. Appl. Supercond., Vol. 21, Issue 3, 12022677, 2011.

[4] Mizuno K., Tanaka M. and Ogata M., "Mechanical Vibration Tests on Real-scale REBCO Coil," Quarterly Report of RTRI, Vol. 59, No. 4, pp. 287-292, 2018.

[5] Mizuno K., Yamashita T., Miyazaki Y., Sakamoto H., Nakao K., Matsui Y. and Dohi T., "Enhance a maximum load of High Temperature Superconducting Magnetic Bearing -New type coil structure can balance low heat with cooling-," Abstracts of CSSJ Conference, Vol. 94, p. 128, 2017 (in Japanese).

\section{Authors}

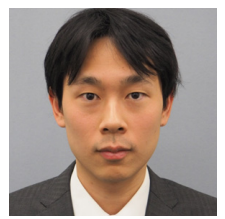

Katsutoshi MIZUNO Dr.Eng.

Assistant Senior Researcher, Cryogenic

Systems Laboratory, Maglev Systems

Technology Division

Research Areas: Superconducting Technology

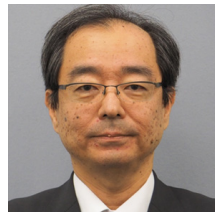

Masafumi OGATA Dr.Eng.

Senior Chief Researcher, Head of Cryogenic

Systems Laboratory, Maglev Systems

Technology Division

Research Areas: Superconducting Technology

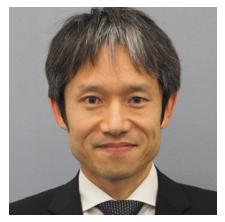

Minoru TANAKA Dr.Eng.

Senior Researcher, Cryogenic Systems

Laboratory, Maglev Systems Technology

Division

Research Areas: Electrical Engineering,

Superconducting Technology 